\title{
Intracisternal Interleukin-1 Receptor Antagonist Prevents Postoperative Cognitive Decline and Neuroinflammatory Response in Aged Rats
}

\author{
Ruth M. Barrientos, Amy M. Hein, Matthew G. Frank, Linda R. Watkins, and Steven F. Maier \\ Department of Psychology and Neuroscience, Center for Neuroscience, University of Colorado at Boulder, Boulder, Colorado 80309
}

To investigate the role of the pro-inflammatory cytokine interleukin-1 $\beta$ (IL-1 $\beta)$ in postoperative cognitive dysfunction (POCD) in aged rats, we used laparotomy to mimic human abdominal surgery in adult (3 months) and aged (24 months) F344/BN rats. We demonstrated that memory consolidation of the hippocampal-dependent contextual fear-conditioning task is significantly impaired in aged but not young rats $4 \mathrm{~d}$ after surgery. Hippocampal-independent auditory-cued fear memory was not disrupted by laparotomy in either age group. The hippocampal-dependent memory impairment was paralleled by elevations of IL-1 $\beta$ in the hippocampus of aged animals 1 and $4 \mathrm{~d}$ after surgery. These findings support our substantial line of previous research showing that aged animals are more vulnerable to cognitive decline after a peripheral immune challenge. In addition, we demonstrated that a single intracisternal administration of interleukin-1 receptor antagonist (IL-1RA; $112 \mu \mathrm{g}$ ) at the time of surgery was sufficient to block both the behavioral deficit and the neuroinflammatory response. Injecting the same dose of IL-1RA peripherally failed to have a protective effect. These data provide strong support for the specific role of central, not peripheral, IL-1 $\beta$ in POCD. Furthermore, the long-lasting presence of IL-1RA in the brain (4 d) compared with in the blood $(<24 \mathrm{~h})$ underscores the value of intracisternal administration of IL-1RA for therapeutic purposes.

\section{Introduction}

The first comprehensive report that older people exhibit cognitive declines after surgery was published over 50 years ago (Bedford, 1955). Patients were described to "never be the same since" the operation. Symptoms varied from being disoriented to having difficulty remembering events, planning, or sustaining attention. Early studies focused on cardiac surgeries, presumably due because of possible hypoxic and/or ischemic episodes during this type of surgery. The depth of anesthesia necessary for these types of surgeries was also presumed to be a contributing factor (Bedford, 1955). This effect is now called postoperative cognitive dysfunction or decline (POCD), and it continues to negatively impact the quality of life of millions each year. There is now clear evidence that noncardiac surgeries (e.g., abdominal and orthopedic) can also produce POCD (Moller et al., 1998; Monk et al., 2008). Moreover, recent studies have found no causal relationship between depth of anesthesia and POCD (Rasmussen et al., 2003; Steinmetz et al., 2010). POCD typically lasts days to weeks, with a small minority of patients exhibiting longer-lasting declines (Abildstrom et al., 2000). Strikingly, the occurrence of POCD at 3 months was associated with an increased risk of death

Received May 6, 2012; revised Aug. 7, 2012; accepted Aug. 28, 2012.

Author contributions: R.M.B., L.R.W., and S.F.M. designed research; R.M.B., A.M.H., and M.G.F. performed research; R.M.B. analyzed data; R.M.B. wrote the paper.

This work was supported by National Institute on Aging Grant R01AG028271 (R.M.B., M.G.F., L.R.W., and S.F.M.). A. M. Hein's present address: Strategic Analysis, 4075 Wilson Boulevard, Suite 200, Arlington, VA 22203.

Correspondence should be addressed to Dr. Ruth M. Barrientos, Department of Psychology and Neuroscience, Campus Box 345, University of Colorado at Boulder, Boulder, C080309-0345. E-mail: ruth.barrientos@colorado.edu. DOI:10.1523/JNEUROSCI.2173-12.2012

Copyright $\odot 2012$ the authors $\quad 0270-6474 / 12 / 3214641-08 \$ 15.00 / 0$ in the first year after surgery (Monk et al., 2008). There is robust evidence that advanced age is the single strongest risk factor for the development of POCD (Moller et al., 1998). Prior histories of cognitive impairment or perioperative events, such as strokes, are additional risk factors for POCD (Monk et al., 2008). Despite much research in this area, a mechanism underlying this agingrelated vulnerability to POCD remains primarily unknown. Much of the difficulty has come from variable definitions of POCD, methodologies in diagnosing POCD, and varied and inconsistent experimental designs across research studies (Rasmussen et al., 2001).

Many of the observations described above are consistent with extensive findings from our laboratory, and others, demonstrating that aged subjects are far more vulnerable to long-term memory declines after a challenging life event than are young adult subjects. This research has shown that aging primes microglial cells, such that a subsequent immune challenge results in an exaggerated inflammatory response in the hippocampus, which in turn produces significant hippocampal-dependent memory deficits (Barrientos et al., 2002, 2006, 2009, 2011; Cunningham et al., 2005; Godbout et al., 2005; Chen et al., 2008; Dilger and Johnson, 2008; Frank et al., 2010a). Importantly, because the peripheral immune system has several modes of communication to the brain, a peripheral challenge (such as surgery) is capable of resulting in de novo production of pro-inflammatory cytokines within the brain (Maier, 2003). And, in the aged organism, this neuroinflammatory response is long lasting (Barrientos et al., 2009).

In the present study, we examined POCD after abdominal surgery in young and aged rats. Furthermore, we examined the 
central versus peripheral role of interleukin-1 in producing the cognitive decline in aged rats after surgery by administering human interleukin-1 receptor antagonist (hIL-1RA) either intracisternally or intraperitoneally at the time of surgery to protect against POCD.

\section{Materials and Methods}

Subjects. Subjects were male F344xBN F1 rats obtained from the National Institute on Aging (Bethesda, MD). On arrival at our facility, aged rats were 24 months old and weighed $\sim 575 \mathrm{~g}$. Young adult rats were 3 months old and weighed $\sim 280 \mathrm{~g}$. All rats were age matched and housed two to a cage [52 length $(\mathrm{L}) \times 30$ width $(\mathrm{W}) \times 21$ height $(\mathrm{H}) \mathrm{cm}$ ]. The animal colony was maintained at $22 \pm 1^{\circ} \mathrm{C}$ on a $12 \mathrm{~h}$ light/dark cycle (lights on at 7:00 A.M.). All rats were allowed ad libitum access to food and water and were given at least 1 week to acclimate to colony conditions before experimentation began. All experiments were conducted in accordance with protocols approved by the University of Colorado Animal Care and Use Committee. All efforts were made to minimize the number of animals used and their suffering.

Surgery. Laparotomy and sham surgeries were performed using aseptic procedures under halothane anesthesia using a previously described method developed as a model of human abdominal exploratory surgery (Martin et al., 2005). The abdominal region was shaved and thoroughly cleaned with $70 \%$ ethanol and surgical scrub. Approximately $0.5 \mathrm{~cm}$ below the lower right rib, a $3 \mathrm{~cm}$ vertical incision was made, penetrating the peritoneal cavity. Wearing sterile latex gloves, the surgeon inserted an index finger up to the second knuckle into the opening and vigorously manipulated the viscera and musculature. Approximately $10 \mathrm{~cm}$ of the intestine was then exteriorized and vigorously rubbed between the surgeon's thumb and index finger for $30 \mathrm{~s}$. The intestines were then placed back into the peritoneal cavity. Sterile chromic gut sutures (3-0, chromic gut, 27 inches, PS-2; Ethicon) were used to suture the peritoneal lining and abdominal muscle in two layers. The skin was closed with surgical staples. To prevent infection, the wound was dressed with Polysporin (Pfizer). Sham-operated rats were anesthetized, and the abdominal area was shaved and cleaned as described above. They remained on halothane for the same amount of time as their surgical counterpart ( $\sim 25 \mathrm{~min}$ ).

Contextual fear conditioning. Either 4 or $12 \mathrm{~d}$ after surgery, rats were taken two at a time from their home cage, and each was placed in a conditioning chamber $(26 \mathrm{~L} \times 21 \mathrm{~W} \times 24 \mathrm{H}, \mathrm{cm})$ made of clear plastic and topped with a wire mesh top. Each chamber was housed inside an ice chest $(54 \mathrm{~L} \times 30 \mathrm{~W} \times 27 \mathrm{H}, \mathrm{cm})$. A speaker, a fan, and two 24 -V DC light bulbs (one white, one red) were mounted on the ceiling of each ice chest. Rats were allowed to explore the chamber for $2 \mathrm{~min}$ before the onset of a $15 \mathrm{~s}$ tone $(76 \mathrm{~dB})$, followed immediately by a $2 \mathrm{~s}$ footshock $(1.5 \mathrm{~mA})$ delivered through a removable floor of stainless steel rods. Each rod was wired to a shock generator and scrambler (Coulbourn Instruments). To assess obvious signs of lethargy or sickness, locomotion was scored during conditioning. Immediately after the termination of the shock, rats were removed from the chamber and returned to their home cage. Seventy-two hours later, all rats were tested for fear of the conditioning context (a hippocampal-dependent task) and for fear of the tone (a hippocampal-independent task). Chambers were cleaned with water and a mild detergent before each animal was conditioned or tested. For the context fear test, rats were placed in the exact context in which they were conditioned, observed for $6 \mathrm{~min}$, and scored for freezing behavior. For the auditory-cued fear test, rats were placed in an altered context (e.g., different shaped and sized chambers, red light, no grid floor) and scored for freezing behavior for $3 \mathrm{~min}$. After the $3 \mathrm{~min}$, the tone was activated, and freezing behavior was scored for an additional $3 \mathrm{~min}$. Freezing is the rat's dominant defensive fear response, and it is a common measure of conditioned fear (Kim and Fanselow, 1992). Freezing was defined as the absence of all visible movement, except for respiration. Every $10 \mathrm{~s}$, each rat was judged as either freezing or active at the instant the sample was taken. Inter-rater reliability exceeded $97 \%$ for all experiments.

Intracisterna magna or intraperitoneal administration of hIL-1RA. To determine whether peripheral or central interleukin-1 $\beta$ (IL-1 $\beta$ ) may be playing a role in disrupting hippocampal-dependent memory after sur- gery in aged rats, separate groups of animals received either a peripheral or central administration of hIL-1RA (112 $\mu$ g per rat; Amgen). Immediately before commencing laparotomy surgery, rats were anesthetized with halothane. For rats receiving the peripheral administration, hIL1RA was injected intraperitoneally in a total volume of $250 \mu \mathrm{l}$. For rats receiving the central administration, the dorsal aspect of the skull was shaved and swabbed with 70\% EtOH. A 27 gauge needle attached via PE50 tubing to a $25 \mu \mathrm{l}$ Hamilton syringe was inserted into the cisterna magna. To verify entry into the cisterna magna, $\sim 2 \mu \mathrm{l}$ of clear CSF was drawn and gently pushed back in and a $3 \mu \mathrm{l}$ total volume of hIL-1RA was administered. An equal volume of sterile saline was injected [intracisterna magna $(\mathrm{icm})]$ into vehicle control animals.

Tissue dissection and blood sampling. To eliminate any possibility that the behavioral manipulations described above may contribute to alterations in brain cytokines, separate groups of animals were used for the protein measurements after surgery. One, four, or 12 days after surgery, animals were given a lethal dose of sodium pentobarbital. Blood was collected from a cardiac puncture, and the rat was transcardially perfused with ice-cold saline $(0.9 \%)$ for 2 min to remove peripheral immune leukocytes from the CNS vasculature. Brains were rapidly extracted and placed on ice, and hippocampi were dissected and rapidly frozen in liquid nitrogen. A sample $(\sim 100 \mathrm{mg})$ of liver was also dissected and frozen. Blood was centrifuged at $10,000 \times g$ at $4^{\circ} \mathrm{C}$ for $10 \mathrm{~min}$, and serum was collected. Tissue and serum samples were stored at -80 until assays were performed.

Detection of rat IL-1 $\beta$ and $h I L-1 R A$ protein. To prepare the tissues for the assays, $0.3 \mathrm{ml}$ (hippocampus) or $1.0 \mathrm{ml}$ (liver) of a sonication buffer containing $50 \mathrm{~mm}$ Tris base and a mixture enzyme inhibitor $(100 \mathrm{~mm}$ amino-n-caproic acid, $10 \mathrm{~mm}$ EDTA, $5 \mathrm{~mm}$ benzamidine $\mathrm{HCl}$, and 0.2 mu phenylmethyl sulfonyl fluoride) was added to each sample. Each tissue was mechanically sonicated for $\sim 20 \mathrm{~s}$ using an ultrasonic cell disrupter (Thermo Fisher Scientific) and centrifuged at $10,000 \times g$ at $4^{\circ} \mathrm{C}$ for $10 \mathrm{~min}$, and supernatants were removed and stored at $4^{\circ} \mathrm{C}$ until ELISA was performed (usually the next day). Bradford protein assays were also performed to determine total protein concentrations in each sample. Levels of IL-1 $\beta$ or hIL-1RA protein were determined using commercially available rat IL- $1 \beta$ or human IL-1RA ELISA kits (R\&D Systems). The assays were performed according to the manufacturer's instructions. Hippocampal samples used for the IL- $1 \beta$ assay were run at a 2 -fold dilution, and liver samples were run at a 10 -fold dilution. Tissue (hippocampal and liver) sonicated samples used for the hIL-1RA assay were run neat. Serum samples for the hIL-1RA assay were diluted as follows: $1 \mathrm{~h}$ (50-fold), $3 \mathrm{~h}$ (10-fold), $24 \mathrm{~h}$ (neat), $4 \mathrm{~d}$ (neat). The concentration of IL- $1 \beta$ or hIL-1RA protein is presented as picograms per $100 \mu \mathrm{g}$ of total hippocampal or liver protein and as picograms per milliliter for serum samples.

\section{Statistical analyses}

Statistical analyses were conducted using StatView version 5.0 and Prism software. One-way and two-way ANOVAs were used, as appropriate. Auditory-cued memory data and serum hIL-1RA data were analyzed using repeated-measures ANOVAs. Where appropriate, StudentNewman-Keuls post hoc tests were conducted to reveal pairwise differences between groups. In cases where only two groups were being compared, two-tailed $t$ tests were used. Statistical significance for all tests was set at $\alpha=0.05$.

\section{Results}

\section{Experiment 1: contextual fear conditioning}

To investigate the influence of a surgery on subsequent memory performance of a contextual fear-conditioning task, adult and aged rats underwent a sham or abdominal surgery $4 \mathrm{~d}$ before conditioning. It is important to note that during conditioning, all groups explored the novel context with equal interest, nearly $100 \%$ of the time, suggesting that none of the rats were overtly sick or lethargic during conditioning ( $p>0.05$; Fig. $1 A$, inset). Long-term memory was tested $3 \mathrm{~d}$ after conditioning. A two-way ANOVA (age $\times$ surgical condition) revealed a significant age $\times$ 


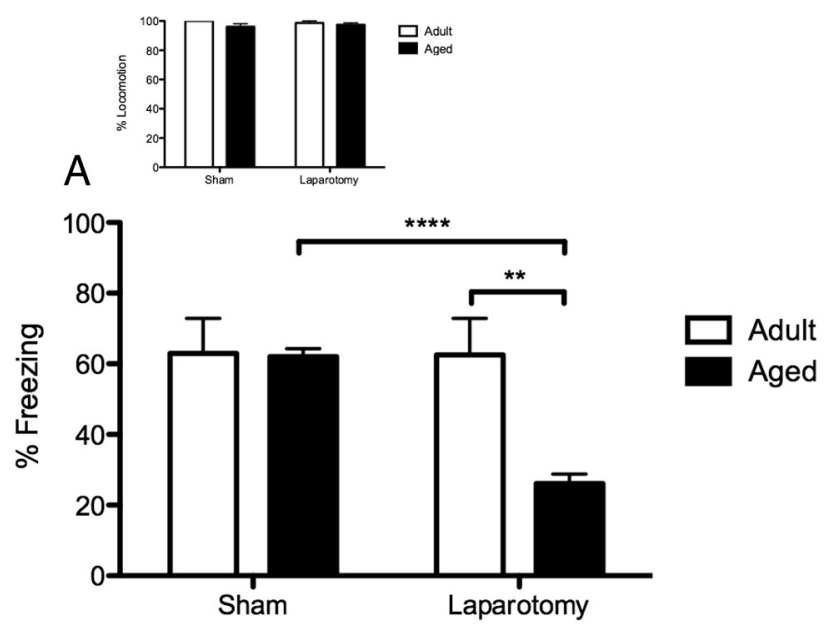

B
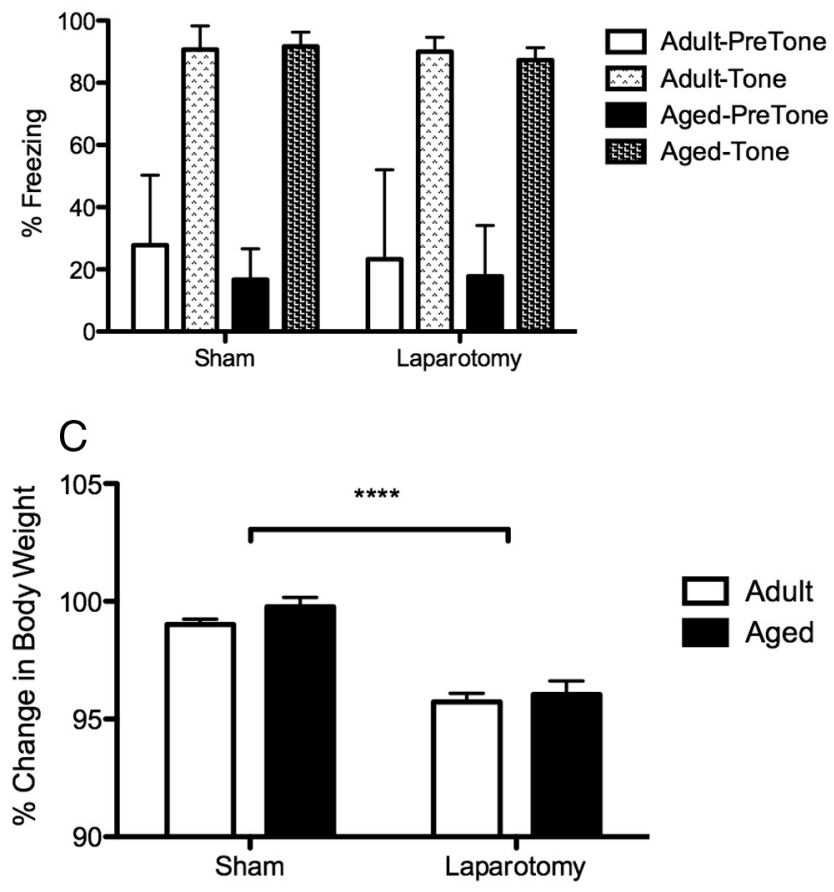

Figure 1. Inset, Locomotion during the conditioning session was nearly $100 \%$ and did not differ between any of the groups. A, Laparotomy $4 \mathrm{~d}$ before contextual fear conditioning produced a significant memory deficit for hippocampal-dependent contextual memory in aged, but not adult, rats. A sham operation did not produce any memory impairments. $\boldsymbol{B}$, There was no effect of surgery, regardless of age, on the hippocampal-independent auditory-cued test. $C$, Laparotomy, regardless of age, produced a significant reduction in percentage of body weight compared with sham-operated groups. ${ }^{* *} p<0.01 ;{ }^{* * * *} p<0.0001$.

surgical condition interaction $\left(F_{(1,18)}=6.77 ; p<0.02\right)$. Post hoc tests confirmed that only aged rats that underwent surgery exhibited significantly reduced freezing in the context compared with aged sham $(p<0.0001)$ and adult operated $(p<0.01)$ rats, indicating a substantial memory deficit in these rats. All other groups performed well and did not differ from one another (Fig. $1 \mathrm{~A})$. For the auditory-cued test, a two-way (age $\times$ surgical condition) repeated-measures (pretone and posttone freezing) ANOVA produced no significant main effect of age $(F<1)$, no significant main effect of surgery $\left(F_{(1,18)}=1.39 ; p>0.05\right)$, and no interaction $F<1$. There was a significant difference between pretone and posttone freezing $\left(F_{(1,18)}=255.04 ; p<0.0001\right)$. Post
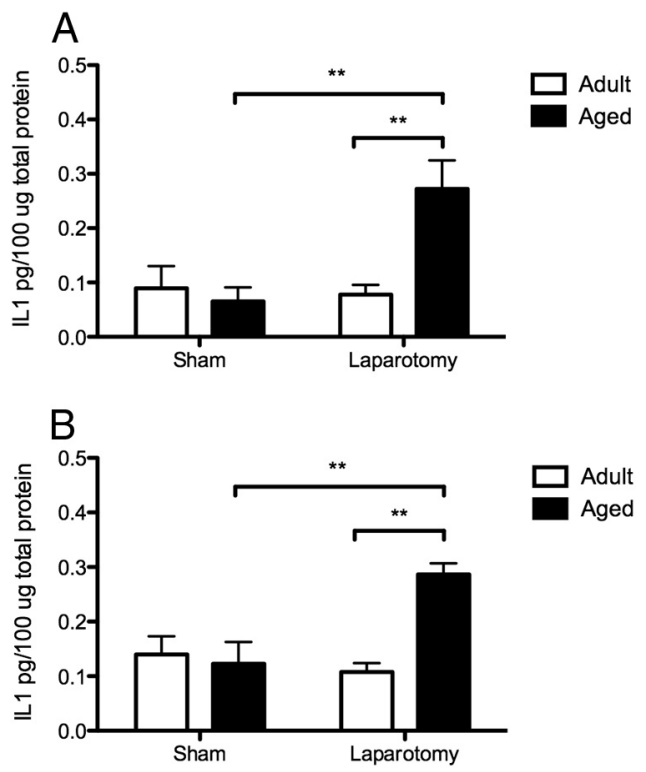

Figure 2. Hippocampal IL-1 $\beta$ protein levels were significantly elevated $24 \mathrm{~h}(\boldsymbol{A})$ and $4 \mathrm{~d}(\boldsymbol{B})$ after laparotomy in aged, but not adult, rats. A sham operation did not produce an elevation in IL- $1 \beta$ at either time point, regardless of age. ${ }^{* *} p<0.01$.

hoc tests revealed that every group froze significantly less before the tone was presented than after, but there was no difference between any of the groups' freezing to the tone (Fig. 1B).

Percentage change in body weight $4 \mathrm{~d}$ after surgery was also examined in these animals. A two-way ANOVA produced a significant effect of surgery $\left(F_{(1,20)}=72.82 ; p<0.0001\right)$ but not of age $\left(F_{(1,20)}=1.66 ; p>0.05\right)$, and there was no interaction $(F<1$; Fig. $1 C)$. These data indicate that undergoing surgery produces a significant weight loss $(\sim 3-4 \%)$. However, it is important to note that aged animals were not more vulnerable than were young adult animals in this regard.

Since adult rats were not cognitively impaired when conditioned $4 \mathrm{~d}$ after surgery, only aged rats were examined with conditioning at $12 \mathrm{~d}$ after surgery. With conditioning at $12 \mathrm{~d}$, aged sham and operated rats performed equally well on the contextual test occurring $3 \mathrm{~d}$ later and were no longer significantly different from one another $\left(t_{(19)}=0.43 ; p>0.05\right.$; data not shown). Not surprisingly, performance on the auditory-cued test was also not impaired between surgery groups at this time point $(F<1)$. There was a significant difference between freezing before the tone and freezing to the tone $\left(F_{(1,19)}=968.87 ; p<0.0001\right)$. Post hoc tests revealed that every group froze significantly less before the tone was presented than after, but there was no difference between any of the groups' freezing to the tone (data not shown).

\section{Experiment 2: hippocampal IL-1 $\beta$}

To assess the levels of IL- $1 \beta$ in the brains of adult and aged rats after a sham or abdominal surgery, rats were killed 1 or $4 \mathrm{~d}$ later. A two-way ANOVA (age $\times$ surgical condition) was conducted. At $1 \mathrm{~d}$ after surgery, there was a significant age $\times$ surgical condition interaction $\left(F_{(1,19)}=8.31 ; p<0.01\right)$. Post hoc tests confirmed that only aged rats that underwent surgery exhibited a significant increase of IL- $1 \beta$ protein compared with adult operated $(p<0.01)$ and aged sham $(p<0.01)$ rats. All other groups did not differ from one another (Fig. $2 A$ ). Similar data were observed $4 \mathrm{~d}$ after surgery. There was a significant age $\times$ surgical condition interaction $\left(F_{(1,19)}=12.32 ; p<0.01\right)$. Post hoc tests at this time point also confirmed that only aged rats that underwent 
surgery exhibited a significant increase in IL- $1 \beta$ protein compared with adult operated $(p<0.0001)$ and aged sham $(p<$ $0.01)$ rats. Again, all other groups did not differ from one another (Fig. $2 B$ ). These data demonstrate that the surgery-dependent elevation of IL- $1 \beta$ in the hippocampus was selective to the aged subjects. Since adult rats were not impaired at $4 \mathrm{~d}$, only aged rats were examined at $12 \mathrm{~d}$ after surgery. At this time point, there was no significant difference between sham and operated aged rats $\left(t_{(10)}=0.85 ; p>0.05\right.$; data not shown $)$.

Experiment 3: intracisterna magna versus intraperitoneal hIL-1RA on contextual fear conditioning

To determine whether IL- $1 \beta$ plays a mediating role in this surgery-induced cognitive effect, we injected a single dose of hIL1RA into aged rats immediately before commencing surgery. We administered hIL-1RA centrally (icm) or peripherally (intraperitoneally) to further distinguish whether peripheral or central signaling of IL- $1 \beta$ was critical to producing the effect. In addition, one control group received a single dose of sterile saline $(\mathrm{icm})$ before surgery, and another control group received an icm dose of IL-1RA before a sham surgery. As before, during conditioning, all groups explored the novel context with equal interest, examining it nearly $100 \%$ of the time, confirming that none of the rats was overtly sick or lethargic during conditioning $(p>0.05$; Fig. $3 A$, inset). A one-way ANOVA of the long-term memory data produced a significant difference between the groups $\left(F_{(3,20)}=\right.$ 7.91; $p<0.001)$. Post hoc tests revealed that freezing levels of vehicle-treated and intraperitoneal hIL-1RA-treated operated rats were not different from each other but were each significantly lower than those of the sham-operated group. These data suggested that administering hIL-1RA via an intraperitoneal route was as ineffective at preventing the impairing effects of surgery as was injecting saline. In contrast, administering hIL-1RA via an icm route produced significantly higher freezing than in the other two surgical groups $(p<0.05)$, and these levels were not different than those of the sham control group. These data clearly demonstrated that blocking central IL- $1 \beta$ signaling was critical to protecting cognitive function against the effects of surgery in aged animals (Fig. 3A). For the auditory-cued test results, a repeatedmeasures ANOVA showed that pretone freezing was significantly lower than freezing to the tone $\left(F_{(3,19)}=538.39 ; p<0.0001\right)$, but freezing to the tone was again confirmed to be unimpaired after surgery and was unaffected by hIL-1RA treatment, regardless of the route of administration $\left(F_{(3,19)}=1.40 ; p>0.05\right.$; Fig. $\left.3 B\right)$.

Percentage change in body weight $4 \mathrm{~d}$ after surgery was also examined in these animals. A one-way ANOVA produced a significant difference between the groups $\left(F_{(3,23)}=3.77 ; p<0.05\right)$. Post hoc tests revealed that each of the surgical groups, regardless of treatment or route of administration, had lower body weights than the sham-operated group ( $p<0.05$; Fig. $3 C)$. Together, these data suggest that blocking central IL-1 signaling was effective at preventing hippocampal-dependent memory deficits after surgery but was not effective at ameliorating the body weight loss resulting from undergoing surgery.

\section{Experiment 4: intracisterna magna versus intraperitoneal hIL-1RA on hippocampal and liver IL-1 $\beta$}

To assess the effects of a central or peripheral injection of hIL1RA just before surgery on levels of IL- $1 \beta$ in the central and periphery nervous systems, aged rats were killed $4 \mathrm{~d}$ after injection/surgery and cytokine levels were examined in the hippocampus and liver. A control group receiving an icm dose of IL-1RA before a sham surgery was also included. In the hippocampus
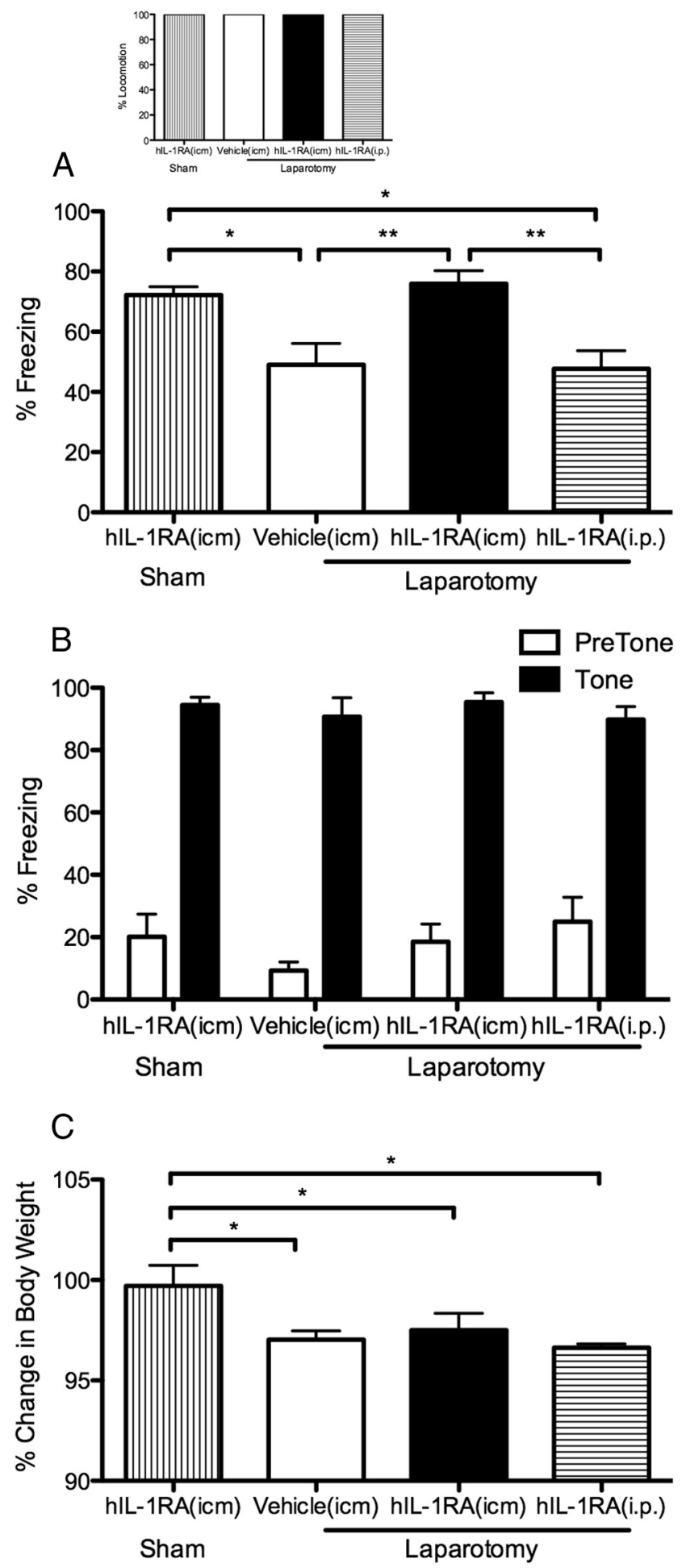

Figure 3. Inset, Locomotion during the conditioning session was nearly $100 \%$ and did not differ between any of the groups. $A$, A single icm injection of hlL-1RA (112 $\mu \mathrm{g})$ administered at the time of surgery prevented the surgery-induced memory consolidation impairments in aged rats $4 \mathrm{~d}$ later. The same dose of hlL-1RA administered intraperitoneally was not effective, as freezing levels did not differ from those of the vehicle-treated group. $\boldsymbol{B}$, Auditory-cued memory was unaffected by surgery or hIL-1RA treatment. $C$, Each of the surgical groups, regardless of treatment or route of administration, had lower body weights compared with the sham-operated group. ${ }^{*} p<0.05$; $^{* *} p<0.01$.

(Fig. 4A), a one-way ANOVA showed a significant difference between the four groups $\left(F_{(3,24)}=8.82 ; p=0.0004\right)$. Post hoc tests revealed that IL- $1 \beta$ levels in the hippocampus of laparotomy plus vehicle-treated and laparotomy plus intraperitoneal hIL-1RA- 

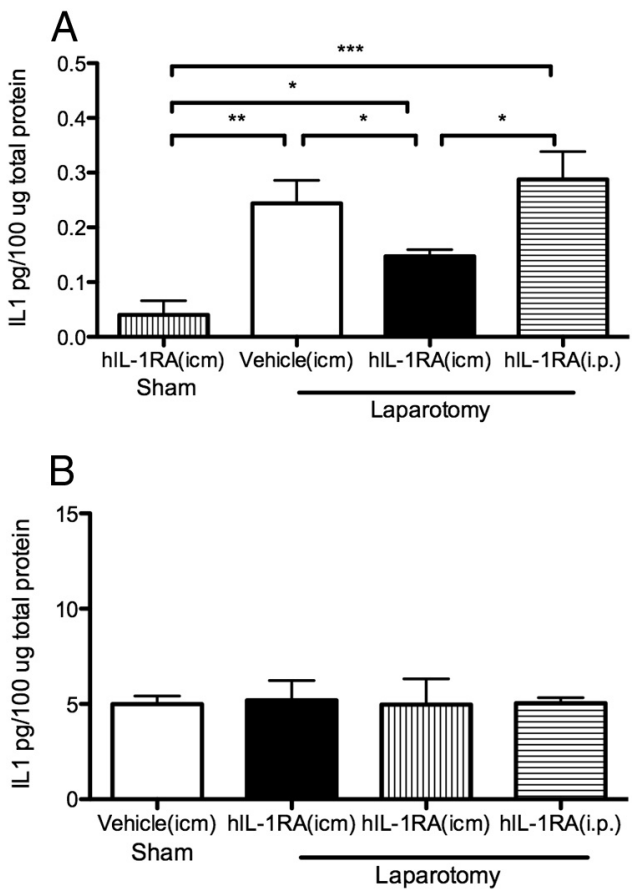

Figure 4. A, A single icm injection of hlL-1RA $(112 \mu \mathrm{g})$ administered at the time of surgery prevented surgery-induced IL-1 $\beta$ protein elevations in the hippocampus in aged rats $4 \mathrm{~d}$ later. The same dose of hlL-1RA administered intraperitoneally was not effective, as IL-1 $\beta$ levels did not differ from those of the vehicle-treated group. $\boldsymbol{B}, \mathrm{IL}-1 \beta$ protein levels in the liver $4 \mathrm{~d}$ after laparotomy were not different across groups. ${ }^{*} p<0.05$; ${ }^{* *} p<0.01$; ${ }^{* * *} p<0.001$.

treated rats were significantly elevated compared with sham plus icm hIL-1RA-treated controls $(p<0.001)$ but were not different from each other $(p>0.05)$, demonstrating that both of these treatments were ineffective at blocking the pro-inflammatory response in the hippocampus produced by surgery. In contrast, IL- $1 \beta$ levels in the hippocampus of laparotomy plus icm hIL1RA-treated rats were significantly lower than laparotomy plus vehicle-treated and laparotomy plus intraperitoneal hIL-1RAtreated rats $(p<0.05$ and $p<0.01$, respectively) and significantly higher than sham plus hIL-1RA-treated controls $(p<0.05)$. It is worth pointing out that our sham control rats here were treated with hIL-1RA, and as expected, their IL- $1 \beta$ levels were reduced to levels lower than noninjected sham animals (see Fig. $2 B$ for reference), so it is not surprising that laparotomy plus icm hIL-1RAtreated rats were not reduced to those levels. In fact, they were reduced to levels comparable with those of noninjected sham controls $(\sim 0.14 \mathrm{pg} / 100 \mu \mathrm{g}$ total protein). These data, together with the behavioral data, suggest that icm administration of hIL1RA just before surgery is effective in significantly reducing proinflammatory cytokine levels in the hippocampus and preventing hippocampal-dependent memory deficits in aged animals. In the liver (Fig. $4 B$ ), a one-way ANOVA showed no significant differences between the four groups $\left(F_{(3,25)}=0.18 ; p>0.05\right)$. First, these data point to the fact that the inflammatory response in the hippocampus far outlasts the inflammatory response in the periphery, suggesting that it is the neuroinflammatory response that is responsible for the cognitive declines in the aged rats. Second, these data strongly support the idea that the protective effects of hIL-1RA are centrally, not peripherally, mediated.

\section{Experiment 5: detection of hIL-1RA in periphery and brain} It has long been known that the terminal half-life of hIL-1RA is $<2 \mathrm{~h}$ when injected peripherally (intravenously) (Granowitz et
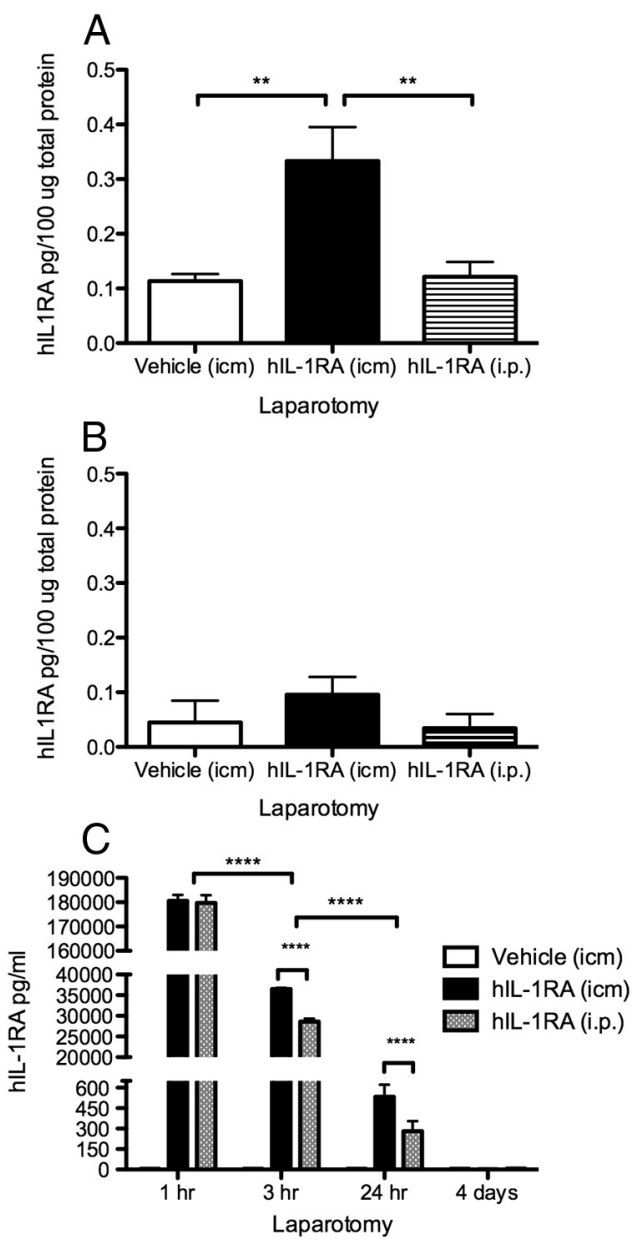

Figure 5. A, In the hippocampus, aged rats that received a single icm injection of hlL-1RA $(112 \mu \mathrm{g})$ at the time of surgery continued to express significantly elevated levels of hIL-1RA compared with background (vehicle) and intraperitoneal IL-1RA-administered levels $4 \mathrm{~d}$ later. intraperitoneal hIL-1RA levels were no different from background levels at this time point. $B$, In the liver, regardless of the route of administration, aged rats that received a single injection of hIL-1RA (112 $\mu \mathrm{g})$ at the time of surgery exhibited levels of hIL-1RA no different from background (vehicle) levels $4 \mathrm{~d}$ later. $C$, In the serum, hll-1RA was highly and equally expressed $1 \mathrm{~h}$ after both routes of administration. At $3 \mathrm{~h}$, hlL-1RA expression levels dropped precipitously (note scale change), with icm-administered levels significantly higher than intraperitonealadministered levels. At $24 \mathrm{~h}$, hlL-1RA expression levels dropped precipitously again (note scale change). At this time point, icm-administered levels continued to be expressed significantly higher than intraperitoneal-administered levels. At $4 \mathrm{~d}$, hlL-1RA expression levels were no longer different than background (vehicle) levels. ${ }^{* *} p<0.01$; ${ }^{* * *} p<0.0001$.

al., 1992). It is less well known whether the kinetics of hIL-1RA are similar within central compartments. If they were similar, it would be puzzling how a single centrally administered dose of hIL-1RA could protect against a long-lasting ( $4 \mathrm{~d})$ neuroinflammatory response produced by surgery. To begin to resolve this conundrum, we examined the levels of hIL-1RA in central and peripheral compartments. It is critical to note that it was human recombinant IL-1RA that was administered in these studies. This is important because hIL-1RA is not only effective in the rat but also allows us to specifically detect the hIL-1RA that was administered apart from endogenous (rat) IL-1RA levels. We measured hIL-1RA in hippocampus, liver, and serum $4 \mathrm{~d}$ after the two routes of administration. In addition, using repeated tail nick blood collections, we measured hIL-1RA in serum at 1,3 , and $24 \mathrm{~h}$ after administration. In the hippocampus (Fig. 5A), hIL1RA was expressed differently in icm hIL-1RA- and intraperito- 
neal hIL-1RA-treated rats $\left(F_{(2,17)}=17.22 ; p<0.0001\right)$. Post hoc tests confirmed that icm hIL-1RA-treated rats exhibited significantly greater levels than those that were administered hIL1-RA intraperitoneally $(p<0.001)$. In the liver (Fig. 5B), hIL-1RA, regardless of the route of administration, was not detected at levels higher than those from vehicle-treated rats $\left(F_{(2,17)}=0.44\right.$; $p>0.05$ ). In the serum (Fig. $5 C$ ), a repeated-measures ANOVA across time points produced a significant drug effect $\left(F_{(2,15)}=\right.$ $6000 ; p<0.0001)$, a significant time point effect $\left(F_{(2,15)}=18,000\right.$; $p<0.0001)$, and a drug $\times$ time point interaction $\left(F_{(2,15)}=4400\right.$; $p<0.0001$ ). Post hoc analysis revealed that at the $1 \mathrm{~h}$ time point, icm hIL-1RA and intraperitoneal hIL-1RA groups were significantly higher than vehicle control levels $(p<0.0001)$ but were not different from each other $(p>0.05)$. At $3 \mathrm{~h}$, there was a precipitous reduction in hIL-1RA levels compared with $1 \mathrm{~h}$ levels $(p<0.0001)$. Furthermore, there was a significant decline in hIL-1RA in intraperitoneal-administered rats compared with icm-administered rats $(p<0.0001)$. At $24 \mathrm{~h}$, there was another precipitous reduction in hIL-1RA levels compared with $3 \mathrm{~h}$ levels $(p<0.0001)$, and levels continued to be detectable above background levels $(p<0.01)$. And again, there was a significant reduction in hIL-1RA in intraperitoneal-administered rats compared with icm-administered rats $(p<0.05)$. At $4 \mathrm{~d}$, hIL1RA expression levels in icm- and intraperitoneal-administered groups were no longer differ from vehicle-treated rats $(p>0.05)$. Together, these data strongly suggest that the degradation rates of IL-1RA in central and peripheral compartments are quite different. In the brain, hIL-1RA persisted for many days, whereas in the periphery it was scarcely detectable at $24 \mathrm{~h}$. Moreover, icm administration of hIL-1RA resulted in reduced degradation in serum and hippocampus compared with intraperitoneal administration.

\section{Discussion}

Young adult rats showed neither a cognitive impairment on memory for contextual fear nor an increase in IL- $1 \beta$ in the hippocampus 1 or $4 \mathrm{~d}$ after surgery. These findings are consistent with what others have found previously (Rosczyk et al., 2008). In contrast, aged rats exhibited a significant deficit in hippocampaldependent memory consolidation $4 \mathrm{~d}$ after surgery. Impairments were no longer present when rats underwent fear conditioning $12 \mathrm{~d}$ after surgery, confirming the human literature that POCD is time limited (Moller et al., 1998; Abildstrom et al., 2000; Monk et al., 2008). Anesthesia alone could not account for these memory impairments in aged animals, as rats that underwent a sham surgery under equal amounts of anesthesia as those that underwent the surgical procedure performed normally and comparably with adult rats. This postoperative cognitive decline was limited to hippocampal-dependent memory. Performance on the auditorycued fear test was unaffected by surgery in aged rats, suggesting that amygdala-dependent memory function was left intact after surgery. These findings are consistent with what others found using an orthopedic surgery model, albeit in young adult mice and a retrograde conditioning paradigm (conditioning occurred just before surgery) (Cibelli et al., 2010). Because we used an anterograde conditioning paradigm (conditioning and test all occurred after surgery), one concern is whether the animals were too sick after surgery to properly sample and encode the environment during acquisition or express fear during the test. This concern is obviated by data demonstrating that all animals explored the conditioning context equally during conditioning and the finding that performance on the auditory-cued test was intact in all animals.
Aged rats exhibited a large elevation of IL- $1 \beta$ in the hippocampus 1 and $4 \mathrm{~d}$ after surgery, a response not observed in sham-operated aged rats or in adult operated rats. IL- $1 \beta$ increases were no longer present $12 \mathrm{~d}$ after surgery. Interestingly, the time course of the IL- $1 \beta$ elevation paralleled the time course of the memory consolidation impairment. Together, these findings suggest that aging organisms are especially vulnerable to memory impairments produced by peripheral immune activation, in this case after an abdominal surgery. These results are consistent with other reports showing that aged subjects are far more vulnerable to such challenges and that these challenges are accompanied by an exaggerated and long-lasting increase of proinflammatory cytokines in the hippocampus, resulting in impaired synaptic plasticity and hippocampal-dependent memory deficits (Barrientos et al., 2006, 2009; 2011; Buchanan et al., 2008; Chen et al., 2008; Abraham and Johnson, 2009; Chapman et al., 2010). One study found that minor abdominal surgery resulted in elevated IL- $1 \beta$ mRNA expression in the hippocampus of aged mice, and this was accompanied by disrupted reversal learning in the Morris water maze (Rosczyk et al., 2008). Several investigations have demonstrated that primed microglia are the cellular source for this amplified neuroinflammatory response (Ye and Johnson, 1999; Cunningham et al., 2005; Henry et al., 2009; Frank et al., 2010a). Others have also found that hippocampaldependent memory is specifically disrupted in aged animals after partial hepatectomy, and this impairment was accompanied by elevations of proinflammatory cytokines and increased microglial activation in the hippocampus, although impairments and neuroinflammatory responses in this study lasted only $1 \mathrm{~d}$ after surgery (Cao et al., 2010).

Although the human literature is variable, the preponderance of data indicates that the majority of older adults that suffer from POCD have symptoms that last a few days to weeks after surgery (Abildstrom et al., 2000). The rate falls dramatically after 1 month after surgery, and it seems to be quite rare for symptoms to last longer than 3 months after a single surgery or without other risk factors (Moller et al., 1998; Monk et al., 2008). With behavioral and neuroinflammatory symptoms robustly exhibited at $4 \mathrm{~d}$ and absent at $12 \mathrm{~d}$, our data here are consistent with these observations. Future studies will examine whether multiple surgeries or multiple immune challenges, such as surgery followed by a bacterial infection, would result in a protracted neuroinflammatory response and longer-lasting POCD symptoms in aged animals. Recent studies using young adult rodents have demonstrated potentiated neuroinflammatory and behavioral responses when animals were subjected to a combination of LPS and surgery (Fidalgo et al., 2011; Hains et al., 2011), suggesting that this "twohit" approach would also be successful in extending POCD symptoms in aged rats.

Blocking central signaling of IL- $1 \beta$ with a single icm administration of hIL-1RA at the time of surgery was sufficient to prevent both the behavioral impairment and the neuroinflammatory response in aged rats. The same dose administered peripherally was not effective in preventing either. These data support the notion that the inflammatory response in the brain, not in the periphery, after surgery is a critical mediator of POCD. Although others have shown that IL- $1 \beta$ plays a role in POCD (Cibelli et al., 2010), we are the first to show that blocking central IL-1 receptors is sufficient to block POCD. These findings are consistent with previous reports showing that blocking IL-1 receptors in the aged brain with hIL-1RA blocks many of the central manifestations resulting from a peripheral innate immune challenge. For instance, long-term memory deficits in aged rats re- 
sulting from a peripheral bacterial infection are associated with reductions in the immediate-early gene, activity-dependent cytoskeletal-associated protein (arc) (Frank et al., 2010b); reductions in theta-burst LTP (Chapman et al., 2010); and reductions in mature BDNF (Cortese et al., 2011). In all of these studies, a single icm administration of hIL-1RA prevented the outcomes.

The long-lasting effects of a single icm administration of hIL1RA seem curious given that the known half-life of IL-1RA is just under $2 \mathrm{~h}$ (Granowitz et al., 1992). Importantly, these findings were derived from studies that administered IL-1RA intravenously. In the present study, we showed that icm- and intraperitoneal-administered hIL-1RA had similar expression levels in the serum and liver. In the serum, our findings support the findings of Granowitz et al. (1992). Levels were quite high $1 \mathrm{~h}$ after injection regardless of the route of administration. At $3 \mathrm{~h}$, levels dropped precipitously. Despite being expressed significantly above background, it is unclear whether these levels are functionally significant. At $24 \mathrm{~h}$, levels were just detectable above background. At $4 \mathrm{~d}$, serum and liver expression levels of hIL-1RA were no longer greater than background levels. Importantly, the different routes of administration resulted in very different expression levels of hIL-1RA in the hippocampus. Levels of hIL1RA $4 \mathrm{~d}$ after administration continued to be significantly expressed in the hippocampus of rats that received the drug centrally $(\mathrm{icm})$ but not those that received it peripherally (intraperitoneal). Levels of hIL-1RA expression in the hippocampus of rats that received the drug peripherally were no different from background levels. Although we did not examine degradative enzymes or metabolism of the drug, we speculate that enzymes that degrade IL-1RA in the circulation may not have equal access to central compartments, therefore allowing longer-lasting effects of the drug when administered centrally. This is important when considering the translational value of the present study. Others have shown that IL-1RA administered peripherally (intraperitoneally) is effective at blocking behavioral and neuroinflammatory responses after surgery; however, the drug needed to be injected repeatedly days before and after surgery (Cibelli et al., 2010). Repeated peripheral injections would be expected to interfere with a peripheral immune function and so would not be a useful prophylactic. Given the increased number of aged humans undergoing surgery, finding an effective and convenient preventative therapy for POCD is of great importance.

\section{References}

Abildstrom H, Rasmussen LS, Rentowl P, Hanning CD, Rasmussen H, Kristensen PA, Moller JT (2000) Cognitive dysfunction 1-2 years after noncardiac surgery in the elderly. ISPOCD Group. International Study of Post-Operative Cognitive Dysfunction. Acta Anaesthesiol Scand 44: $1246-1251$.

Abraham J, Johnson RW (2009) Consuming a diet supplemented with resveratrol reduced infection-related neuroinflammation and deficits in working memory in aged mice. Rejuvenation Res 12:445-453.

Barrientos RM, Higgins EA, Sprunger DB, Watkins LR, Rudy JW, Maier SF (2002) Memory for context is impaired by a post context exposure injection of interleukin-1 beta into dorsal hippocampus. Behav Brain Res 134: 291-298.

Barrientos RM, Higgins EA, Biedenkapp JC, Sprunger DB, Wright-Hardesty KJ, Watkins LR, Rudy JW, Maier SF (2006) Peripheral infection and aging interact to impair hippocampal memory consolidation. Neurobiol Aging 27:723-732.

Barrientos RM, Frank MG, Hein AM, Higgins EA, Watkins LR, Rudy JW, Maier SF (2009) Time course of hippocampal IL-1 beta and memory consolidation impairments in aging rats following peripheral infection. Brain Behav Immun 23:46-54.

Barrientos RM, Frank MG, Crysdale NY, Chapman TR, Ahrendsen JT, Day HE, Campeau S, Watkins LR, Patterson SL, Maier SF (2011) Little exer- cise, big effects: reversing aging and infection-induced memory deficits, and underlying processes. J Neurosci 31:11578-11586.

Bedford PD (1955) Adverse cerebral effects of anaesthesia on old people. Lancet 269:259-263.

Buchanan JB, Sparkman NL, Chen J, Johnson RW (2008) Cognitive and neuroinflammatory consequences of mild repeated stress are exacerbated in aged mice. Psychoneuroendocrinology 33:755-765.

Cao XZ, Ma H, Wang JK, Liu F, Wu BY, Tian AY, Wang LL, Tan WF (2010) Postoperative cognitive deficits and neuroinflammation in the hippocampus triggered by surgical trauma are exacerbated in aged rats. Prog Neuropsychopharmacol Biol Psychiatry 34:1426-1432.

Chapman TR, Barrientos RM, Ahrendsen JT, Maier SF, Patterson SL (2010) Synaptic correlates of increased cognitive vulnerability with aging: peripheral immune challenge and aging interact to disrupt theta-burst latephase long-term potentiation in hippocampal area CA1. J Neurosci 30:7598-7603.

Chen J, Buchanan JB, Sparkman NL, Godbout JP, Freund GG, Johnson RW (2008) Neuroinflammation and disruption in working memory in aged mice after acute stimulation of the peripheral innate immune system. Brain Behav Immun 22:301-311.

Cibelli M, Fidalgo AR, Terrando N, Ma D, Monaco C, Feldmann M, Takata M, Lever IJ, Nanchahal J, Fanselow MS, Maze M (2010) Role of interleukin-1beta in postoperative cognitive dysfunction. Ann Neurol 68: $360-368$.

Cortese GP, Barrientos RM, Maier SF, Patterson SL (2011) Aging and a peripheral immune challenge interact to reduce mature brain-derived neurotrophic factor and activation of TrkB, PLC $\gamma 1$, and ERK in hippocampal synaptoneurosomes. J Neurosci 31:4274-4279.

Cunningham C, Wilcockson DC, Campion S, Lunnon K, Perry VH (2005) Central and systemic endotoxin challenges exacerbate the local inflammatory response and increase neuronal death during chronic neurodegeneration. J Neurosci 25:9275-9284.

Dilger RN, Johnson RW (2008) Aging, microglial cell priming, and the discordant central inflammatory response to signals from the peripheral immune system. J Leukoc Biol 84:932-939.

Fidalgo AR, Cibelli M, White JP, Nagy I, Maze M, Ma D (2011) Systemic inflammation enhances surgery-induced cognitive dysfunction in mice. Neurosci Lett 498:63-66.

Frank MG, Barrientos RM, Watkins LR, Maier SF (2010a) Aging sensitizes rapidly isolated hippocampal microglia to LPS ex vivo. J Neuroimmunol 226:181-184.

Frank MG, Barrientos RM, Hein AM, Biedenkapp JC, Watkins LR, Maier SF (2010b) IL-1RA blocks E. coli-induced suppression of Arc and long-term memory in aged F344xBN F1 rats. Brain Behav Immun 24:254-262.

Godbout JP, Chen J, Abraham J, Richwine AF, Berg BM, Kelley KW, Johnson RW (2005) Exaggerated neuroinflammation and sickness behavior in aged mice following activation of the peripheral innate immune system. FASEB J 19:1329-1331.

Granowitz EV, Porat R, Mier JW, Pribble JP, Stiles DM, Bloedow DC, Catalano MA, Wolff SM, Dinarello CA (1992) Pharmacokinetics, safety and immunomodulatory effects of human recombinant interleukin-1 receptor antagonist in healthy humans. Cytokine 4:353-360.

Hains LE, Loram LC, Taylor FR, Strand KA, Wieseler JL, Barrientos RM, Young JJ, Frank MG, Sobesky J, Martin TJ, Eisenach JC, Maier SF, Johnson JD, Fleshner M, Watkins LR (2011) Prior laparotomy or corticosterone potentiates lipopolysaccharide-induced fever and sickness behaviors. J Neuroimmunol 239:53-60.

Henry CJ, Huang Y, Wynne AM, Godbout JP (2009) Peripheral lipopolysaccharide (LPS) challenge promotes microglial hyperactivity in aged mice that is associated with exaggerated induction of both proinflammatory IL-1beta and anti-inflammatory IL-10 cytokines. Brain Behav Immun 23:309-317.

Kim JJ, Fanselow MS (1992) Modality-specific retrograde amnesia of fear. Science 256:675-677.

Maier SF (2003) Bi-directional immune-brain communication: implications for understanding stress, pain, and cognition. Brain Behav Immun $17: 69-85$

Martin TJ, Kahn WR, Eisenach JC (2005) Abdominal surgery decreases food-reinforced operant responding in rats: relevance of incisional pain. Anesthesiology 103:629-637.

Moller JT, Cluitmans P, Rasmussen LS, Houx P, Rasmussen H, Canet J, 
Rabbitt P, Jolles J, Larsen K, Hanning CD, Langeron O, Johnson T, Lauven PM, Kristensen PA, Biedler A, van Beem H, Fraidakis O, Silverstein JH, Beneken JE, Gravenstein JS (1998) Long-term postoperative cognitive dysfunction in the elderly ISPOCD1 study. ISPOCD investigators. International Study of Post-Operative Cognitive Dysfunction. Lancet 351:857-861.

Monk TG, Weldon BC, Garvan CW, Dede DE, van der Aa MT, Heilman KM, Gravenstein JS (2008) Predictors of cognitive dysfunction after major noncardiac surgery. Anesthesiology 108:18-30.

Rasmussen LS, Larsen K, Houx P, Skovgaard LT, Hanning CD, Moller JT (2001) The assessment of postoperative cognitive function. Acta Anaesthesiol Scand 45:275-289.

Rasmussen LS, Johnson T, Kuipers HM, Kristensen D, Siersma VD, Vila P,
Jolles J, Papaioannou A, Abildstrom H, Silverstein JH, Bonal JA, Raeder J, Nielsen IK, Korttila K, Munoz L, Dodds C, Hanning CD, Moller JT (2003) Does anaesthesia cause postoperative cognitive dysfunction? A randomised study of regional versus general anaesthesia in 438 elderly patients. Acta Anaesthesiol Scand 47:260-266.

Rosczyk HA, Sparkman NL, Johnson RW (2008) Neuroinflammation and cognitive function in aged mice following minor surgery. Exp Gerontol $43: 840-846$.

Steinmetz J, Funder KS, Dahl BT, Rasmussen LS (2010) Depth of anaesthesia and post-operative cognitive dysfunction. Acta Anaesthesiol Scand 54:162-168.

Ye SM, Johnson RW (1999) Increased interleukin-6 expression by microglia from brain of aged mice. J Neuroimmunol 93:139-148. 